\title{
La Terapia Ocupacional comunitaria para la promoción del arte y la cultura: reflexiones desde el proyecto Tienda Cultural
}

Community-based Occupational Therapy for the promotion of art and culture: reflections from the project Tienda Cultural

A Terapia Ocupacional comunitária para a promoção da arte e da cultura: reflexões a partir do projeto Tenda Cultural

Carla Regina Silva ${ }^{1}$

Isadora Cardinalli

Marina Sanches Silvestrini ${ }^{3}$

Ana Carolina da Silva Almeida Prado 4

Leticia Ambrosio ${ }^{5}$

Rúbia Diana da Mota ${ }^{6}$

Izamara Pereira Paterra ${ }^{7}$

Jennifer Cristine Costa de Oliveira ${ }^{8}$

Marco Antonio Liu Targa Mancini ${ }^{9}$

\footnotetext{
1 Terapeuta ocupacional. Magíster en Educación. Doctora en Educación. Profesora, Universidade Federal de São Carlos. San Carlos, Brasil. carlars@ufscar.br fiD http://orcid.org/0000-0002-7079-8340

2 Terapeuta ocupacional. Especialista en Terapia Ocupacional, Arte, Salud y Cultura. Magíster y doctoranda en Terapia Ocupacional, Universidade Federal de São Carlos. San Carlos, Brasil. isadora.cardinalli@gmail.com iD https://orcid.org/0000-0001-8918-3162

${ }^{3}$ Terapeuta ocupacional. Especialista en Gestión Cultural y Economía Creativa. Magíster en Terapia Ocupacional. Centro de Rehabilitación Pediátrica Primeros Pasos. San Carlos, Brasil. marinassilvestrini@gmail.com iD https://orcid.org/0000-0002-2696-6650

${ }^{4}$ Terapeuta ocupacional. Magíster en Terapia Ocupacional. Hospital de Servicio Público Municipal. San Pablo, Brasil. almeidaprado.ahto@gmail.com (iD) https://orcid.org/0000-0002-4964-0450

${ }^{5}$ Terapeuta ocupacional. Magíster y doctoranda en Terapia Ocupacional, Universidade Federal de São Carlos. San Carlos, Brasil. leambrosio.to@gmail.com iD https://orcid.org/0000-0003-0705-6309

${ }^{6}$ Terapeuta Ocupacional. Consultório na Rua, Secretaria Municipal de Saúde. San Bernardo del Campo, Brasil. rubiadmota@gmail.com iD https://orcid.org/0000-0002-0984-4395

${ }^{7}$ Terapeuta Ocupacional. Residente, Universidad de São Paulo. Maestrante en Terapia Ocupacional, Universidade Federal de São Carlos. San Carlos, Brasil. izamarapereirapaterra@gmail.com iD https:// orcid.org/0000-0001-5320-2755

${ }^{\mathbf{8}}$ Becaria del equipo del proyecto Tienda Cultural. San Bernardo del Campo, Brasil. jennifer.c.oliveira@gmail.com (iD) https://orcid.org/0000-0002-9415-2069

${ }^{9}$ Pedagogo. Profesor, Academia Galpão 4. San Pablo, Brasil. marcoaltmm@gmail.com iD https:// orcid.org/0000-0002-3070-854X
} 


\title{
RESUMEN
}

Se presenta el proyecto de extensión universitaria Tienda Cultural: una ventana abierta para el arte, la ciencia y la ciudadanía, y las reflexiones derivadas de su implementación. Su objetivo fue promover, a partir del compromiso ético y político de la Terapia Ocupacional, la accesibilidad, la ciudadanía y la participación sociocultural a través de acciones artísticas, culturales y educativas. Fue una práctica de Terapia Ocupacional en el ámbito de la cultura desde una perspectiva comunitaria crítica y comprometida con la transformación social. La gestión cultural fue coordinada entre equipos transdisciplinarios y comunidades, y la programación se realizó en territorios periféricos de una ciudad mediana brasileña. Se incluyeron talleres artístico-culturales y educativos, exposiciones, cine, espectáculos con artistas y producciones culturales centradas en las demandas, los intereses y la producción artística de cada comunidad. Se generaron reflexiones sobre la cultura como derecho, el rol de la Terapia Ocupacional comunitaria y cultural, y la función social de la universidad pública. La Terapia Ocupacional y la cultura pueden contribuir a promover la diversidad, el reconocimiento y el acceso a la cultura; asimismo, a ampliar la participación social y la ciudadanía en estrecha colaboración con, para y desde las comunidades que enfrentan procesos de desigualdad y exclusión.

\section{PALABRAS CLAVE}

derechos culturales, ciudadanía, diversidad cultural, acción comunitaria, servicio universitario

\begin{abstract}
This article presents the university extension project "Cultural Store: an open window for art, science, and citizenship" and the reflections derived from its implementation. Based on Occupational Therapy's ethical and political commitment, the objectives were to promote accessibility, citizenship, and socio-cultural participation through artistic, cultural and educational activities. The project was an occupational therapy practice within a cultural environment from a critical community perspective, committed to social transformation. Cultural management was coordinated between transdisciplinary teams and communities, and the cultural programming was carried out in peripheral areas from a mid-sized Brazilian city. The programming included socio-cultural and educational workshops, exhibitions, cinema, shows with artists, and cultural productions focused on each community's demands, interests, and artistic production. The results generated reflections on culture as a right, the role of community and cultural Occupational Therapy, and the social function of the public university. Occupational Therapy and cultural activity can contribute to promote cultural diversity, recognition, and access. They also expand social participation and citizenship for communities experiencing exclusion and unequal processes, by intimately collaborating with, for, and within them.
\end{abstract}




\section{KEY WORDS}

cultural rights, citizenship, cultural diversity, community action, study service (higher education)

\section{RESUMO}

Apresenta-se o projeto de extensão universitária "Tenda Cultural: janela aberta para arte, ciência e cidadania", assim como as reflexões derivadas de sua implementação. A partir do compromisso ético político da Terapia Ocupacional, seu objetivo foi promover acessibilidade, cidadania e participação sociocultural por meio de ações artísticas, culturais e educativas. Foi uma prática da Terapia Ocupacional no campo da cultura a partir de uma perspectiva critica comunitária e comprometida com a transformação social. A gestão cultural foi realizada entre equipes transdisciplinares e comunidades e a programação cultural em territórios periféricos de uma cidade de médio porte brasileira. A programação incluiu oficinas artístico-culturais e educativas, exposições, cine, espetáculos com artistas e produções culturais baseadas nas demandas, interesses e produção artística de cada comunidade. Os resultados demonstraram reflexões da cultura como direitos, o papel da Terapia Ocupacional comunitária e cultural e a função social da universidade pública. A Terapia Ocupacional e a cultura podem contribuir significativamente para a promoção da diversidade e o reconhecimento e acesso à cultura, assim como ampliar a participação social e a cidadania em consonância com, para e a partir das comunidades que enfrentam cotidianamente processos de desigualdades e exclusões.

\section{PALAVRAS-CHAVE}

direitos culturais, cidadania, diversidade cultural, ação comunitária, serviço universitário

\section{Introducción}

A partir de la década de 1970, el desarroIlo de la Terapia Ocupacional en Brasil estuvo marcado por debates éticos y políticos que incidieron en una fuerte construcción de prácticas sociales y comunitarias en el campo de la salud colectiva, en la asistencia social, la educación y el trabajo. En este sentido, el compromiso de la profesión con la transformación social se ha promovido en función de los campos de práctica y los diferentes aportes teóricos y metodológicos.

Según Vinzon et al. (2020), no hay consenso en las teorizaciones sobre comunidad propuestas por terapeutas ocupacionales, por lo que a menudo son controvertidas. No obstante, Palacios (2017) recuerda que los seres humanos son necesariamente ocupacionales y comunitarios. Así, las prácticas comunitarias de Terapia Ocupacional ocurren en territorios concretos, en la cotidianidad de las comunidades, del barrio, de la población, de la familia y sus relaciones (Palacios, 2013).

En este artículo se presentan las experiencias y reflexiones que surgieron en el desarrollo del proyecto Tienda Cultural: ventana abierta al arte, la ciencia y la ciudadanía. Esta práctica de Terapia Ocupacional planteó un abordaje en el ámbito de la cultura desde una 
perspectiva comunitaria crítica y comprometida con la transformación social. Por esto, en el proyecto se consideraron los sujetos-colectivos, sus actividades humanas y las ocupaciones colectivas de manera situada y contextualizada en tiempo y espacio socio-histórico como fenómenos complejos envueltos en dimensiones políticas, económicas, culturales, sociales, ambientales y afectivas de forma integrada e interdependiente (Silva et al., 2017). Lo anterior teniendo en cuenta que "el reconocimiento y [la] reflexión crítica sobre la realidad cultural, socioeconómica y política del contexto permiten aprehender el objeto de estudio de la Terapia Ocupacional" (Tenorio et al., 2016, p. 467). Además se comprendió la ocupación como proceso de toma de consciencia, liberación y transformación social acorde con el momento histórico en el que tiene lugar.

De este modo, en el proyecto se plantearon acciones en el campo de la cultura a partir del compromiso ético y político basado en la accesibilidad, la ciudadanía y la participación sociocultural. Tal como lo plantea Palacios (2017):

Una intervención comunitaria desde perspectivas críticas supone una comprensión de la realidad social dada por las estructuras económicas de producción, culturales, materiales y simbólicas; busca que los participantes de los procesos tomen conciencia de su posición en la estructura de poder, de sus intereses y necesidades, y de la relación entre ambos aspectos; se aspira al cambio en el orden social, rompiendo la relación de sometimiento y de dominación. (p. 78)

Es en este sentido que Zango (2017) afirma que la Terapia Ocupacional co- munitaria es un proceso continuo, dinámico y participativo cuyo objetivo es que la comunidad adquiera mayor protagonismo. Por eso las prácticas pueden ser en, con y desde la comunidad.

\section{Terapia Ocupacional y cultura}

La Declaración de Posición sobre Diversidad y Cultura de la Federación Mundial de Terapeutas Ocupacionales (WFOT, 2010) está basada en cuatro principios esenciales: la diversidad; los derechos humanos y la inclusión; el lenguaje y la competencia; las actitudes, conocimientos y habilidades. Aun así, la relación entre Terapia Ocupacional y cultura implica una serie de debates, interpretaciones y posibilidades en el seno de la profesión, como los planteados por Beagan (2015), Dorneles y Lopes (2016), Gonçalves et al. (2017) y Silvestrini et al. (2019).

En este artículo priorizamos la cultura como campo de práctica de la Terapia Ocupacional, teniendo en cuenta las relaciones intrínsecas de esta actuación con aspectos macropolíticos y económicos, así como con la micropolítica de las ocupaciones, de las actividades humanas y de las relaciones humanas, atravesadas por las diferentes dimensiones de la cultura. En esta línea, entendemos la cultura desde una perspectiva integrada e implicada en:

Las dimensiones simbólicas de los sujetos en sus ocupaciones, identidades, pertenencias y diversidades; como en sus expresiones y producciones materiales e inmateriales; en las dimensiones económicas, en el enfrentamiento de las problemáticas 
sociales y de desigualdad, en la relación con el mercado de trabajo y otras cuestiones derivadas del sistema de producción capitalista, y en las dimensiones de ciudadanía interesadas en las demandas de acceso, promoción y derechos culturales para todos. (Silva et al., 2017 , p. 112)

Consideramos que reconocer la cultura como un campo de práctica para la Terapia Ocupacional permite ampliar sus posibilidades de actuación, sus intersecciones, repertorios y posibilidades de construir relaciones con las personas, las comunidades, los territorios y los acontecimientos para impulsar los derechos, la ciudadanía y la participación cultural (Silva et al., 2017; Silvestrini, 2019).

Dorneles y Lopes (2016) proponen la ciudadanía y la diversidad cultural como conceptos clave en el abordaje de Terapia Ocupacional, ya que permiten "romper con los procesos de exclusión causados por las visiones tradicionales eurocentristas, elitistas y políticas de asimilación en el contexto de la globalización neoliberal" (p. 112). A su vez, Silva et al. (2017) consideran las prácticas de Terapia Ocupacional y cultura como posibilidades para reflexionar, investigar y construir conocimiento disciplinar en interconexión con otros campos, al tiempo que posibilitan la vivencia de experiencias profesionales sensibles que propician la innovación en la formación y en la intervención sobre realidades y contextos sociales concretos.

Además, valorar las singularidades y pluralidades que se expresan culturalmente en los procesos de transformación y empoderamiento individuales y colectivos tiene como eje la defensa de una actuación sensible. Esta considera la producción de subjetividades y aboga por la denuncia de las cicatrices que deja la lucha contra un régimen jerárquico y opresor, al tiempo que muestra las potencialidades de acción y las fuerzas de resistencia para vivir y crear en la vida cotidiana (Silva et al., 2017).

Lo anterior parte de considerar que las y los terapeutas ocupacionales son sujetos políticos que promueven una práctica igualmente política. Esto implica "tener una concepción del mundo, conocer la situación que nos circunda, comprender la realidad, esto es, tener la capacidad de ver y comprender el mundo donde estamos inmersos, poner posición, criterio y puntos de vista frente a ello" (García, 2016, p. 86).

En esta línea, defender la cultura como derecho humano es esencial en la valoración de la diversidad. Así, es clave reconocer la ciudadanía y el desarrollo económico y simbólico como ejes de las expresiones plurales y diversas representadas en los modos de vida, las actividades humanas, las ocupaciones cotidianas, las tradiciones, la ancestralidad de un pueblo y sus diferentes manifestaciones, las cuales deben ser preservadas y valoradas.

Silvestrini et al. (2019) afirman que la Terapia Ocupacional en el campo de la cultura considera esta como un derecho en todas sus dimensiones, de manera que reconoce su capacidad y su potencial para crear símbolos, significados y múltiples posibilidades de ser, estar y relacionarse. También, como generadora de productos y valores que contribuyen al desarrollo local de manera sostenible e inclusiva. Al respecto, Silva et al. (2017) agregan:

El terapeuta ocupacional se revela como un profesional articulado entre la 
fruición, la promoción, la producción y la gestión cultural para promover de forma amplia e integrada las diferentes dimensiones de la cultura -simbólica, económica y ciudadana- implicadas en las ocupaciones de las personas y de los colectivos, en la promoción de sus propias culturas y en defensa de la ciudadanía, de la pertenencia, de la diversidad y de los derechos sociales y humanos. (p. 116)

La investigación de Silvestrini et al. (2019), realizada con terapeutas ocupacionales que desarrollaron prácticas en este campo, presenta tres dimensiones relacionadas: gestión cultural; creación y producción cultural, y fruición y participación cultural. Repecto a la gestión cultural, las autoras consideran que esta:

Busca estrategias para organizar y calificar actividades, proyectos y acciones inventivas en el ámbito cultural, considerando sus aspectos organizativos, estructurales y económicos con el fin de incentivar el fortalecimiento de construcciones culturales más diversas, económicamente colaborativas, creativas y sostenibles. (Silvestrini et al., 2019, p. 104)

Por lo tanto, el abordaje de la Terapia Ocupacional en el ámbito de la cultura implica considerar herramientas de la gestión alineadas de forma sensible con el universo de la cultura y, por tanto, incorporar conceptos técnicos como los de planificación estratégica, plan de comunicación, metodología de evaluación y articulación de redes, personas y procesos.

En ese contexto, este artículo aborda la implementación de un proyecto de gestión cultural planteado desde la perspectiva de la Terapia Ocupacional comunitaria crítica, que abarca nuestro compromiso ético y político con las personas y las comunidades con las cuales trabajamos (Lima, 2003; Pino et al., 2015; Silva et al., 2017; Palacios, 2017; Zango, 2017).

\section{Descripción del proyecto}

El proyecto Tienda Cultural: ventana abierta al arte, la ciencia y la ciudadanía se llevó a cabo con las comunidades de territorios periféricos de São Carlos (en el estado de São Paulo), una ciudad mediana de Brasil. Tuvo como objetivos promover el acceso de esas comunidades a actividades artísticas y culturales; colaborar con la divulgación científica y la promoción de acciones educativas, y contribuir con la promoción, la profesionalización, el reconocimiento y el fortalecimiento de grupos artísticos locales.

Se trató de un proyecto de extensión universitaria articulado con las demandas sociales de las comunidades, de manera que tuvo como principios la democratización del saber académico y la búsqueda de respuestas a demandas socioculturales desde la experiencia práctica y el ejercicio ético para la formación de estudiantes de grado y posgrado.

La inversión en acciones culturales y educativas diversificadas para las comunidades de territorios periféricos, dada su situación de vulnerabilidad social o riesgo, fue considerada una propuesta de inclusión y de participación sociocultural. En tal sentido, esas acciones favorecen la participación activa y protagónica de la comunidad, que con frecuencia encuentra barreras de acceso a 
los centros de producción y circulación del arte y la cultura. Al mismo tiempo, pueden ampliar las posibilidades y condiciones de vida; minimizar las relaciones injustas desde estrategias dialógicas, y contribuir con la politización, el protagonismo de la comunidad y al estar-en-común. Como lo describen Pino et al. (2015), "La importancia de estar-en-común es encontrar junto con la comunidad momentos que faciliten conversaciones/acciones, muestras de solidaridad, espacios donde la cultura pueda ser un puente que devele nuestra realidad" (p. 18).

\section{Planificación y ejecución}

El proyecto se realizó gracias a la asociación entre la Universidade Federal de São Carlos -con apoyo de la Pro-Rectoría de Extensión y el Grupo de Actividades Humanas y Terapia Ocupacional- y la organización no gubernamental Instituto Cultural Ventana Abierta; contó además con el patrocinio del Banco de Brasil.

La Tienda Cultural: ventana abierta al arte, la ciencia y la ciudadanía tuvo tres ediciones, cada una realizada con una comunidad periférica diferente. En cada una de ellas se ofertaron actividades artísticas, culturales y educativas durante la mañana, tarde y noche de tres días consecutivos (viernes, sábado y domingo). Se eligieron estos días para facilitar el acceso de la población, ya que son principalmente trabajadores, trabajadoras y estudiantes.

Las tres comunidades fueron seleccionadas de acuerdo con características predominantes como el distanciamiento del área central de la ciudad (más desarrollada socioeconómicamente); la escasez de inversiones, actividades artístico-culturales y otras acciones derivadas de políticas públicas. En definitiva, barrios con mayores índices de vulnerabilidad social. Estos territorios están conformados mayoritariamente por una clase trabajadora con baja escolaridad, que se desempeña en trabajos informales y presta servicios vinculados a la construcción y a la limpieza. Allí habita un gran número de familias provenientes de otras regiones de Brasil, quienes vinieron en búsqueda de mejores condiciones de vida. Aunque son territorios periféricos, sus habitantes cuentan con servicios públicos esenciales como atención primaria en salud, educación y asistencia social.

Si bien las administraciones gubernamentales en el pasado hicieron inversiones para realizar presentaciones teatrales en plazas públicas en estas comunidades, los recortes en el sector cultural han generado que en los últimos años no se haya planteado ninguna acción similar. Esto genera malestar en las y los habitantes y artistas de la comunidad, así como en otros sectores preocupados por la ausencia de actividades culturales significativas, como la organización no gubernamental y la universidad involucradas en este proyecto. En consecuencia, la búsqueda de financiación privada para desarrollarlo fue clave, ya que no había apoyo del Gobierno local.

El equipo de trabajo estuvo conformado por profesionales de servicios comunitarios vinculados a la salud y la asistencia social, mujeres consideradas líderes de la comunidad, profesionales de la organización no gubernamental con actuación en las comunidades, un 
gestor de comunicación, dos fotógrafos y el grupo de la Universidad. En este último participaron una coordinadora docente del curso de Terapia Ocupacional, dos terapeutas ocupacionales que trabajaron como agentes culturales y veinte monitoras y monitores graduados de diferentes carreras (Terapia Ocupacional, Pedagogía, Psicología, Música, Biología, Ingeniería Civil, Imagen y Sonido, Letras, Lingüística y Ciencias Sociales). Así, el equipo reunió a profesionales y estudiantes de diferentes áreas del conocimiento y constituyó una actuación transdiscipli- nar, es decir, amplió los límites disciplinarios de la práctica común.

El proceso de planificación estratégica (Guindani et al., 2011) se llevó a cabo durante los tres meses previos a la realización de los eventos y mediante el sistema de gestión por objetivos. En este se tuvieron en cuenta el tiempo, la infraestructura, el presupuesto y los factores limitantes. En la figura 1 y la tabla 1 se identifican y describen los elementos constitutivos de la planificación estratégica y su interconexión.

Figura 1. Sistema de gestión por objetivos del proyecto Tienda Cultural

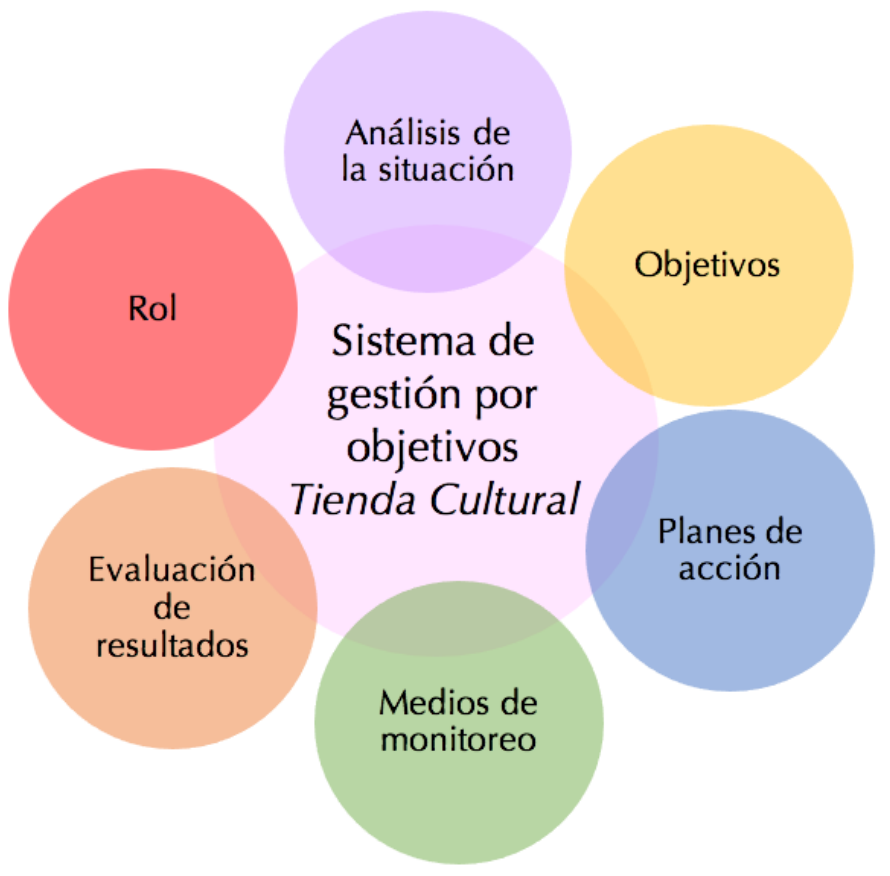

Fuente: elaboración propia. 
Tabla 1. Descripción del sistema de gestión por objetivos del proyecto Tienda Cultural

\begin{tabular}{|c|c|}
\hline Paso & Descripción \\
\hline Rol & $\begin{array}{l}\text { Oportunidad de convivencia con las diversas producciones artístico-culturales y } \\
\text { educativas en comunidades periféricas, desde la cultura ciudadana. }\end{array}$ \\
\hline $\begin{array}{l}\text { Análisis de la } \\
\text { situación }\end{array}$ & $\begin{array}{l}\text { Mapeo de las comunidades y actividades artístico-culturales por medio del trabajo } \\
\text { de campo para la identificación de ofertas y producciones existentes. Realización } \\
\text { de alianzas con la comunidad, profesionales y gestores de servicios públicos y del } \\
\text { tercer sector que actúan en el territorio para resaltar la importancia de las acciones } \\
\text { comunitarias e involucrar a toda la comunidad en ocupaciones significativas. }\end{array}$ \\
\hline Objetivos & $\begin{array}{l}\text { Fortalecimiento de la producción, la circulación y el consumo de actividades } \\
\text { artísticas y culturales. } \\
\text { Promoción y aproximación de actividades académicas y científicas a las } \\
\text { comunidades periféricas. }\end{array}$ \\
\hline $\begin{array}{l}\text { Planes de } \\
\text { acción }\end{array}$ & $\begin{array}{l}\text { - Conformación y consolidación del equipo gestor y ejecutor. } \\
\text { - Asociación entre proyectos universitarios, grupos aficionados y profesionales de } \\
\text { la ciudad y de la región. } \\
\text { - Gestión financiera. } \\
\text { - Trabajo de campo para reconocer las demandas locales (participación en } \\
\text { reuniones, promoción de la participación de las lideresas comunitarias; mapeo } \\
\text { - de artistas y grupos artísticos de cada comunidad). } \\
\text { - } \text { - } \text { - Curadiverollo del plan de comunicación de las actios de comunididades artísticas. } \\
\text { - Definición y logística de la programación. } \\
\text { - Recepción de artistas y profesionales que coordinan talleres y otras actividades. } \\
\text { - Desarrollo de la logística con respecto a la infraestructura (aspectos legales, de } \\
\text { seguridad, de manutención, técnicos, de limpieza, baños químicos) y la } \\
\text { alimentación del equipo. }\end{array}$ \\
\hline $\begin{array}{l}\text { Medios de } \\
\text { monitoreo }\end{array}$ & $\begin{array}{l}\text { - Gestión financiera (presupuestos, patrocinio, contrapartidas). } \\
\text { - } \quad \text { Logística (plazos de las actividades a desarrollar). } \\
\text { - } \quad \text { Alianzas con el sector cultural de la ciudad y de la Universidad. }\end{array}$ \\
\hline $\begin{array}{l}\text { Evaluación de } \\
\text { los resultados }\end{array}$ & $\begin{array}{l}\text { - Elaboración de informes de evaluación y revisión de la contrapartida para el } \\
\text { - } \text { Eatrocinador. } \\
\text { - } \text { los participantes. } \\
\text { - Registro y memoria de las actividades. } \\
\text { - Presentación del balance financiero. } \\
\text { - Realización de pagos e informe de rendición de cuentas. }\end{array}$ \\
\hline
\end{tabular}

Fuente: elaboración propia. 
La programación se planteó desde diferentes frentes conectados con el fomento del arte, la cultura, la ciencia y la ciudadanía: talleres artístico-culturales, exposiciones, presentaciones y espectáculos de artes escénicas, musicales y cine. Estos se desarrollaron de la siguiente manera: uno o dos talleres socioeducativos matinales; después, una pausa para almorzar; luego, dos a cuatro talleres y presentaciones escénicas en la tarde; una sesión de cine al anochecer, y tres presentaciones o espectáculos nocturnos. Además, una exposición fotográfica diaria. También se incluyó la estrategia de interacción con el público, facilitando intercambios respecto a las actividades culturales.

Se cumplieron todos los procedimientos administrativos y legales para obtener la autorización del Ayuntamiento Municipal, los bomberos y la seguridad pública. Se contrataron un equipo de limpieza y de seguridad, servicios de infraestructura, iluminación, camerinos, escenario, generadores de energía, equipo técnico de sonido, baños, sillas, agua y materiales para señalización y emergencia. En estos contratos se dio prioridad a las personas y grupos de la propia comunidad. En todos los momentos del proyecto se mantuvo el diálogo y la negociación con la comunidad, los órganos oficiales de la ciudad y el patrocinador. Además, se realizaron actividades de comunicación y divulgación a través de televisión, radio, medios impresos, periódicos, redes sociales y comunicación directa en cada territorio.

Se resalta la importancia de las reuniones organizativas del equipo ejecutor, basadas en el plan estratégico, y del trabajo comunitario de planificación conjunta, en el marco de los cuales se realizaron encuentros de experimentación de recursos y actividades de integración y formación del equipo. Por ejemplo, se llevaron a cabo encuentros de sensibilización para el trabajo con comunidades en situaciones de vulnerabilidad y violencia, manejar situaciones de conflicto y compartir con los equipos de trabajo.

Para cada edición del proyecto se realizaron reuniones de evaluación entre los miembros del equipo de trabajo y con el público durante la ejecución de las actividades. Para esto, se les invitaba a responder preguntas sobre su participación y los sentimientos movilizados; también se grabaron algunos videos con sus relatos.

En este proyecto los conceptos de democratización y diversidad cultural se extendieron a las estrategias de participación de todas las personas involucradas. Para definir los temas y presentaciones el equipo se respetaron las observaciones, deseos, demandas y sugerencias aportadas por la comunidad. Para esa escucha activa, además de reuniones con las lideresas locales y profesionales de servicios comunitarios, se realizaron encuestas para la construcción colectiva y corresponsable de los eventos. Adicionalmente, todas las actividades tuvieron una base dialógica que garantizó la interacción y la participación constantes, lo que fomentó el intercambio de conocimientos y prácticas culturales.

\section{Resultados y reflexiones a partir de la implementación del proyecto}

Al contrastarlo con sus objetivos, es posible afirmar que el proyecto Tienda Cultural alcanzó resultados positivos. Las estrategias de sentir-pensar-hacer con fueron esenciales para la apropia- 
ción de las actividades por parte de las comunidades.

En cada edición de la Tienda Cultural se desarrollaron entre 11 y 14 talleres artístico-culturales de hasta dos horas de duración cada uno; estos tuvieron un carácter práctico e interactivo para la divulgación de contenidos específicos. Se realizaron entre seis y ocho presentaciones artísticas o espectáculos de danza, teatro, circo, música o performance; entre uno y dos conciertos musicales; tres sesiones de cine; una a dos exposiciones fotográficas, de artes visuales o literarias, y muestras interactivas de educación y ciencia. En total, se logró llegar a cerca de 2.000 personas.

Se fomentaron discusiones y reflexiones con las y los participantes acerca de la participación política y de cuestiones como el género, la violencia, el consumo de drogas, entre otros asuntos que surgieron durante la construcción y planificación con la comunidad.

Las acciones desarrolladas también se analizaron a partir de categorías identificadas en las evaluaciones procedimentales realizadas con el equipo. A continuación se presentan las principales reflexiones en tres de ellas: cultura como derecho; Terapia Ocupacional en la gestión de proyectos; función social de la universidad y el proyecto de extensión como estrategia.

\section{Cultura como derecho}

De acuerdo con los artículos 215 y 216 de la Constitución Ciudadana del Brasil, "El Estado garantizará a todos el pleno ejercicio de los derechos cultura- les y el acceso a las fuentes de la cultura nacional, y apoyará y alentará la valoración y la difusión de las manifestaciones culturales" (Presidencia de la República de Brasil, 1988). Sin embargo, esa perspectiva de la política cultural como bien público ejercido por cada persona y en su comunidad, y que debería ser accesible para todos y todas es reciente y contraria a la idea de la cultura como producto a ser consumido en una lógica de mercado capitalista y neoliberal.

En ese sentido, tenemos presente que en el proceso histórico de las políticas culturales la inversión pública ha sido insuficiente en Brasil y en América Latina. Dada la importancia de la cultura para el desarrollo de cualquier sociedad (Unesco, 1982), esta situación plantea un debate que necesita avanzar y que está relacionado con el debilitamiento de las políticas y los derechos sociales y con las dinámicas de inversión que priorizan unos aspectos en detrimento de otros (Rubim, 2007; Baquero, 2011; Durand, 2013).

Esa disputa, también ideológica, es constante y perpetúa una idea de cultura como superflua y privilegio en una consigna individual y meritocrática. Así, resulta necesario buscar medios para construir prácticas basadas en la cultura como derecho y en el ejercicio de la ciudadanía cultural.

En el ámbito cultural, la experiencia posibilitó una diversidad de actividades y propició el contacto con técnicas y expresiones artísticas, muchas de las cuales eran desconocidas o inaccesibles; de esta manera se amplió la accesibilidad cultural para buena parte del público. Así lo describieron las personas participantes con variadas narrativas: 
"Nunca antes había visto una orquesta", "No imaginé aprender circo o graffiti", "Nunca escuché sobre danza circular", entre otras. También se ofrecieron acciones significativas y atractivas para cada comunidad, cuidadosamente identificadas durante el trabajo de campo previo y con la ayuda, principalmente, de las lideresas en los territorios (figura 2).

Cada edición tuvo como propósito proponer la cultura como un derecho a ser ejercido y producido por todas las personas en su pluralidad y potencia. Los talleres, experimentaciones y presentaciones proporcionaron al público el contacto con diferentes expresiones artísti- cas, el aprendizaje de técnicas y saberes diversos, así como la posibilidad de despertar intereses y experiencias plurales.

Los temas transversales a estas actividades fueron el sexismo, la violencia de género, los prejuicios, la inclusión, la preservación ambiental y la participación social y política. En consecuencia, se realizaron debates colectivos sobre, por ejemplo, la condición de la mujer en esas comunidades. Estos propiciaron reflexiones sobre costumbres sexistas $y$ machistas como el cuidado de la casa impuesto desde temprano a las niñas y la privación de determinadas ocupaciones.

Figura 2. Presentaciones de artes escénicas en ediciones de la Tienda Cultural
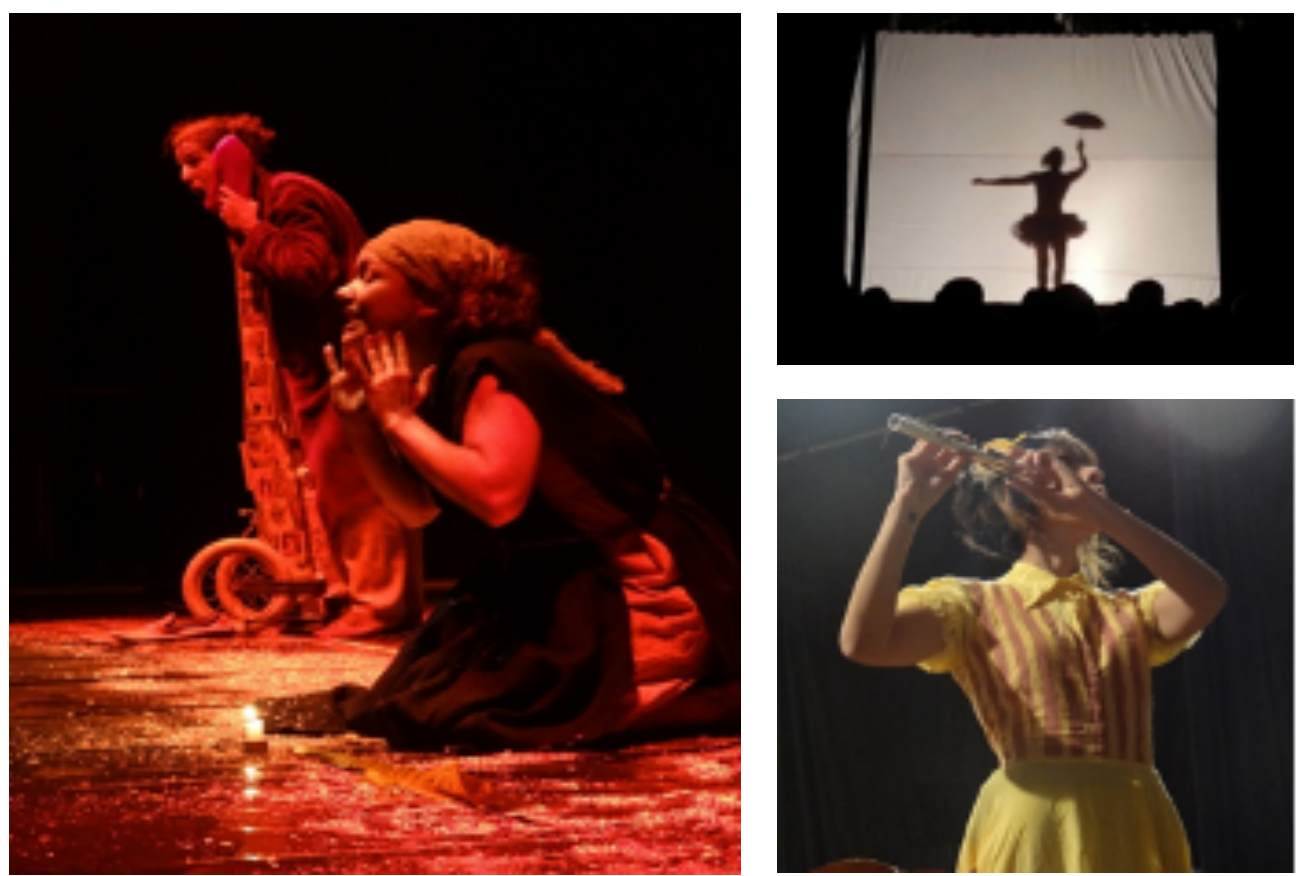

Fuente: elaboración propia a partir de los registros fotográficos de integrantes del equipo de trabajo, realizados con el consentimiento de las y los artistas. 
La programación de actividades culturales fue diversa y heterogénea y valoró la participación de artistas y grupos de la comunidad y de la región, ya que estos no suelen estar presentes en el circuito cultural comercial.

En las actividades planteadas hubo una amplia participación de la comunidad. El público infantil y juvenil fue el que más activamente participó de las actividades y la ocupación del espacio, sobre todo durante el día. En las actividades nocturnas era más común la presencia de personas jóvenes, adultas, adultas mayores y familias. Resalta la presencia de mujeres involucradas con el liderazgo en sus comunidades, quienes trabajan activamente para alcanzar visibilidad y aportar a la solución de los problemas locales.

\section{Terapia Ocupacional en la gestión de proyectos}

La gestión cultural incluye diversas especificidades y principios que pueden articularse con los saberes de la Terapia Ocupacional, ya que como campo conceptual y práctico presenta herramientas y estrategias interesantes y disponibles para profesionales de diferentes disciplinas que actúan en el sector de la cultura.

La experiencia del proyecto Tienda Cultural fue una oportunidad para reconocer y denominar prácticas de la Terapia Ocupacional y de la cultura, permitiéndonos integrar los saberes y haceres de los diferentes campos, en conexión con los territorios y las comunidades.

En el desarrollo del proyecto fue necesario apropiar algunos conceptos técnicos como: plan de comunicación; planificación estratégica; metodología de evaluación; articulación de personas, redes y procesos; organización estructural, ejecutiva y logística. Así mismo, los pasos o etapas de la gestión: planear, compartir, mapear y evaluar durante la ejecución de cada edición.

Planificar, mapear y evaluar en el contexto cultural hace referencia a relacionar saberes diferentes, interculturales. Desde la Terapia Ocupacional comunitaria y crítica se busca la valoración, el protagonismo y la expresión de la vida, las actividades y ocupaciones significativas; de tradiciones, memorias, símbolos y modos de vida.

Esta mirada nos conduce a reflexionar sobre el impacto de las acciones realizadas por el proyecto en la esfera microsocial, donde las actividades artísticas y culturales pueden potenciar propuestas de inclusión y de participación sociocultural de grupos y comunidades en situación de vulnerabilidad social o riesgo y, al mismo tiempo, favorecer la actuación en la comunidad (Canclini, 2003).

Así, sobre la integración de la Terapia Ocupacional y la cultura, defendemos una gestión cultural que asocie métodos, técnicas, procesos y herramientas del campo de la gestión, alineados de forma sensible y estética con el universo de la cultura, e implicada con los derechos y la participación social. Es decir, una gestión cultural que valore los principios y el compromiso ético, político y ancestral, que son fundamentales en la práctica de la Terapia Ocupacional comunitaria crítica. Como lo señala Turino (2010):

Gestión es definición de política, implica la toma de posición, de campo ideológico y no puede ser confundida 
con un proceso neutro. Una gestión competente y comprometida debe presentar una conducta pública coherente en la que los conceptos y las políticas presentados a la sociedad permitan la construcción de consensos, transformando sus logros en conquistas de la ciudadanía. (p. 200)

Nuestra forma de producir la gestión cultural buscó estrategias vinculadas a la perspectiva de los abordajes propios de la Terapia Ocupacional. En este sentido, en la organización y la planificación de las actividades, los proyectos y las acciones creativas se otorgó valor a las formas sensibles y al respeto de la diversidad, se promovió la inclusión y la emancipación para la fruición y la creación cultural, actuando de forma dialógica y colaborativa para priorizar a la propia comunidad (Freire, 1979, 1987).

Concordamos con Vinzón y Oliveira (2018) en que la Terapia Ocupacional tiene un compromiso colectivo, ético, político y sensible con los aconteceres de las personas y sus comunidades, y en que nos sea posible creer en la utopía de construir con nuestras acciones otro mundo posible: más justo, inclusivo y humano.

\section{Función social de la universidad y el proyecto de extensión como estrategia}

La posibilidad de llevar a cabo proyectos de investigación y de extensión desde diferentes áreas del saber ha facilitado intercambios enriquecedores entre sectores académicos y comunitarios. A través de este proyecto se realizaron acciones que involucraron a la ciencia, la cultura, la salud, la educa- ción y el medio ambiente a través del teatro, talleres, ciclismo, capoeira, producción de huertas, entre otras.

Todas las actividades ofrecidas fueron gratuitas y pensadas para atender a un público diverso, en el intento de valorar las diversidades e incluir a la mayor cantidad posible de personas de todas las edades. Se constituyó un espacio de composiciones, de compartir, de integración e intercambios. De esta forma, fue posible resignificar las nociones de cultura y educación como derechos de todos los ciudadanos y ciudadanas, y disminuir las distancias entre los saberes y las prácticas de la comunidad y de la universidad.

El proyecto Tienda Cultural proporcionó a las y los académicos involucrados la oportunidad de transitar entre diferentes áreas del conocimiento, experimentar estrategias frente a los desafíos de la práctica, ejercer su compromiso ético y político e interpelarse desde las adversidades presentes en las comunidades. Esto implicó considerar el propósito de este proyecto y reconocerse como parte de la comunidad. Lo anterior revela la complejidad de enseñar a partir de las demandas sociales que emergen en el intercambio con las comunidades y la profundidad de la formación crítica y reflexiva que se basa en los saberes nutridos por la experiencia práctica y comprometida.

Es importante señalar que las acciones involucraron y estimularon desplazamientos sensibles y afectivos entre el equipo, la comunidad, las y los talleristas y artistas (Silva, 2014). Las evaluaciones de quienes participaron destacan el potencial del proyecto en la formación ética y la sensibilización frente a la 
diferencia y la diversidad. Para esto es necesario comprender los distintos modos de vida, otras realidades y cómo lo contradictorio hace parte de los procesos democráticos y potencia la pluralidad cuando se trabaja en colectivo.

La extensión universitaria, en este sentido, se comprendió como un elemento central de la formación académica de las y los terapeutas ocupacionales, pues la integración entre práctica y teoría favorece la enseñanza. Además, planear y desarrollar acciones con la comunidad, a partir de las demandas emergentes, revela la extensión como función social de la universidad.

El conocimiento generado parte de la inmersión reflexiva de alumnos, alumnas y de la misma comunidad en la vida cotidiana de las comunidades, valorando los deseos, las potencialidades, las conexiones y los recursos disponibles. De esta forma, el conocimiento se con-parte de forma respetuosa en la experiencia práctica dialógica y se nutre de los intercambios de saberes y sentimientos, del manejo de situaciones y de los aprendizajes construidos a partir de las propias contradicciones vividas en los proyectos sociales y culturales.

\section{Conclusiones}

El proyecto Tienda Cultural se preocupó por conocer y reconocer el potencial, los intereses y los saberes de las comunidades; por promover el acceso y la fruición cultural de nuevas experiencias en los campos de las artes y la educación. Por esto, se planteó visibilizar y valorar a las y los artistas y culturas locales. Los resultados de su implementación en tres comunidades generan reflexiones sobre la cultura como derecho, el rol de la Terapia Ocupacional comunitaria crítica y de la cultura, y sobre la función social de la universidad pública.

A través de la Tienda Cultural fue posible realizar actividades educativas y de divulgación científica, estimular procesos creativos y experimentar una serie de vivencias que provocaron desplazamientos sensibles para sostener nuevos deseos y posibilidades de proyectos culturales contrahegemónicos. También permitió integrar saberes y construcciones en diferentes formatos, espacios y estrategias para la democratización de la cultura.

En lo que se refiere a la Terapia Ocupacional comunitaria crítica para la promoción de la cultura, pudimos constatar la importancia de contar con profesionales comprometidos con la gestión, la producción, la promoción y la fruición cultural. Esto posibilitó apropiaciones y transformaciones individuales y colectivas, plurales y singulares, así como valorar a la comunidad y dar un especial protagonismo a las lideresas, los y las artistas y talleristas.

La actuación de terapeutas ocupacionales en el campo de la cultura, en especial en esta experiencia de gestión cultural, contribuyó significativamente a la construcción y la implementación de acciones socioculturales, a la defensa de los derechos y al respeto por la diversidad en la búsqueda de ampliar la participación, el ejercicio de la ciudadanía y la accesibilidad cultural. Ejercer este papel implica un compromiso colectivo, ético, político y sensible para establecer nuevas creaciones y resistencias necesarias. 
Asimismo, la transdisciplinariedad influyó positivamente en el desarrollo del proyecto. Posibilitó una mayor autonomía, contribuyó a la integración del equipo y permitió resolver rápidamente los problemas. Adicionalmente, el proyecto aportó al crecimiento personal y profesional de quienes participamos en él, a la horizontalidad en las relaciones, al intercambio y a la apropiación de conocimientos y técnicas de las diversas áreas involucradas.

Esta experiencia refuerza además la importancia de la universidad pública, gratuita y responsable en el desarrollo de las comunidades donde está inmersa, que ofrezca formación práctica, comprometida e implicada.

La principal limitación del proyecto está en su brevedad, esto es, en las dificultades para su sostenibilidad. La realización de otras ediciones depende de diferentes factores, principalmente la financiación. Como se ha mencionado, en el contexto actual el Estado no prioriza inversiones en la cultura. Por otra parte, el acceso a la inversión privada depende de lograr vender una propuesta convincente y competitiva en la lógica del mercado, la ganancia y la oferta cultural dominante.

No obstante, esta experiencia nos invita a construir prácticas y reflexiones desde la Terapia Ocupacional comunitaria crítica que consideren los aportes de la participación en actividades culturales, entendiendo que puede contribuir significativamente a promover el deseo de la diversidad, a ampliar la participación social y la ciudadanía, y a fomentar la accesibilidad cultural con, para y desde las comunidades que enfrentan vulnerabilidades cotidianamente.

\section{Agradecimentos}

A todas las personas que ocuparon la Tienda Cultural y contribuyeron en su realización.

A la Pro-Rectoría de Extensión de la Universidade Federal de São Carlos, al Instituto Cultural Ventana Abierta y al Banco de Brasil por su apoyo, participación y patrocinio. 


\section{Referencias}

Baquero, M. (2011) Cultura(s) política(s) e democracia no século XXI na América Latina. UFRGS.

Beagan, B.L. (2015). Approaches to culture and diversity: A critical synthesis of occupational therapy literature. Canadian Journal of Occupational Therapy, 82(5), 272-282. https://doi. org/10.1177/0008417414567530

Presidencia de la República de Brasil (1988, 5 de octubre). Constituição da República Federativa do Brasil de 1988. https://www. senado.leg.br/atividade/const/con1988/ CON1988 05.10.1988/CON1988.asp

Canclini, N.G. (2003). Reconstruir políticas de inclusão na América Latina. En Unesco, Políticas culturais para o desenvolvimento: uma base de dados para a cultura (pp. 21-38). Unesco.

Dorneles, P. y Lopes, R.E. (2016) Cidadania e diversidade cultural na pauta das políticas culturais. Cadernos de Terapia Ocupacional da UFSCar, 24(1), 173-183. https://doi. org/10.4322/0104-4931.ctoARF0669

Durand, J.C. (2013). Política cultural e economia da cultura. SESC.

Federación Mundial de Terapeutas Ocupacionales [WFOT] (2010). Declaración de posicionamiento: diversidad y cultura. WFOT. https://www.wfot.org/checkout/1856/1760

Freire, P. (1987). Pedagogia do oprimido. Paz e Terra.

Freire, P. (1979) Educação e mudança. Paz e Terra.
García, S. (2016). Terapeuta ocupacional: sujeto político. Revista Ocupación Humana, 16 (1), 84-90. https://doi. org/10.25214/25907816.16

Gonçalves, M.V., Costa S.L. y Takeiti, B.A. (2017). Terapia ocupacional e cultura: atravessamento, recurso ou campo de atuação. RevisbraTO, 1(5), 538-555. https://revistas.ufri.br/index.php/ribto/article/viewFile/10078/pdf

Guindani, R.A., Martins, S.T. y Sertek, P. (2011). Administração e planejamento estratégico. Ibpex.

Lima, E. (2003). Desejando a diferença: considerações acerca das relações entre os terapeutas ocupacionais e as populações tradicionalmente atendidas por estes profissionais. Revista de Terapia Ocupacional da Universidade de São Paulo, 14(2), 6471. https://doi.org/10.11606/issn.2238-6149. v14i2p64-71

Palacios, M. (2013). Ocupación colectiva, sentido de comunidad y bienestar psicosocial. En Colegio de Terapeutas Ocupacionales de Chile, 50 años de terapia ocupacional en Chile: prácticas, epistemología y realidades locales. 1er Congreso Chileno de Terapia Ocupacional, Tomo 1 (pp. 143-159). On Demand.

Palacios, M. (2017). Reflexiones sobre las prácticas comunitarias: aproximación a una terapia ocupacional del sur. Revista Ocupación Humana, 17(1), 73-88. https://doi. org/10.25214/25907816.157

Pino, J., Ceballos, M. y Sepúlveda, R. (2015). Terapia ocupacional comunitaria crítica: diálogos y reflexiones para iniciar una propuesta colectiva. Revista Electrónica 
de Terapia Ocupacional Galicia - TOG, 12(22), 1-20. http://www.revistatog.com/ num22/pdfs/colab3.pdf

Rubim, A.A.C. (2007). Políticas culturais no Brasil: tristes tradições. Galáxia. Revista do Programa de Pós-Graduação em Comunicação e Semiótica, 1(13), 101-113. https://revistas.pucsp.br/galaxia/article/ view/1469

Silva, C.R. (Org.). (2014). Direitos humanos para diversidade: construindo espaços de arte, cultura e educação. São Jorge.

Silva, C.R., Cardinalli, I., Silvestrini, M.S., Farias, A.Z., Almeida, A. C. S., Ambrosio, L., Oliveira, M.T. y Paula, B. M. (2017). La terapia ocupacional y la cultura: miradas a la transformación social. Revista Chilena de Terapia Ocupacional. 17(1), 109-117. https:// doi.org/10.5354/0719-5346.2017.46383

Silvestrini, M.S. (2019). Terapia ocupacional e cultura: uma curadoria de tessituras entre práticas, políticas, diversidade e direitos [tesis de maestría, Universidade Federal de São Carlos]. Repositorio UFSCar. https:// repositorio.ufscar.br/handle/ufscar/11260

Silvestrini, M.S., Silva, C.R. y Almeida Prado, A.C.S. (2019). Occupational therapy and culture: ethical-political dimensions and resistances. Cadernos Brasileiros de Terapia Ocupacional, 27(4), 929-940. https:// doi.org/10.4322/2526-8910.ctoarf1727

Tenorio, L., Satizabal, M. y Vergara, C.H. (2016). El aporte de terapia ocupacional desde las capacidades. Un encuentro con las comunidades. En S. Simó, A. Guajardo, F. Oliver, S. Galheigo y S. García (eds.), Terapias ocupacionales desde el sur: derechos humanos, ciudadanía y participación (pp. 455-470). USACH.
Turino, C. (2010). Ponto de cultura: o Brasil de baixo para cima. Anita Garibaldi.

Unesco. (1982). Conferencia Mundial sobre las Políticas Culturales. Informe final. Unesco. http://unesdoc.unesco.org/images/0005/000525/052505sb.pdf

Vinzón, V., Allegretti, M. y Magalhães L. (2020). Um panorama das práticas comunitárias da terapia ocupacional na América Latina. Cadernos Brasileiros de Terapia Ocupacional, 28(2), 600-620. https://doi. org/10.4322/2526-8910.ctoAR1891

Vinzón, V. y Oliveira, I.A.L. (2018). Fórum Social Mundial: catalizador de luchas y resistencias. Revista Argentina de Terapia Ocupacional. 4(1), 52-56. https:// revista.terapia-ocupacional.org.ar/RATO/ 2018jul-cronica.pdf

Zango, I. (2017). Terapia ocupacional comunitaria. Editorial Síntesis. 